\title{
Dimensional regularization of the gravitational interaction of point masses
}

\author{
Thibault Damour ${ }^{1)}$, Piotr Jaranowski ${ }^{2)}$ and Gerhard Schäfer ${ }^{3)}$ \\ 1) Institut des Hautes Études Scientifiques, 91440 Bures-sur-Yvette, France \\ 2) Institute of Theoretical Physics, University of Białystok, Lipowa 41, 15-424 Białystok, Poland \\ 3) Theoretisch-Physikalisches Institut, Friedrich-Schiller-Universität, Max-Wien-Platz 1, 07743 Jena, Germany
}

\begin{abstract}
We show how to use dimensional regularization to determine, within the Arnowitt-Deser-Misner canonical formalism, the reduced Hamiltonian describing the dynamics of two gravitationally interacting point masses. Implementing, at the third post-Newtonian (3PN) accuracy, our procedure we find that dimensional continuation yields a finite, unambiguous (no pole part) 3PN Hamiltonian which uniquely determines the heretofore ambiguous "static" parameter: namely, $\omega_{s}=0$. Our work also provides a remarkable check of the perturbative consistency (compatibility with gauge symmetry) of dimensional continuation through a direct calculation of the "kinetic" parameter $\omega_{k}$, giving the unique answer compatible with global Poincaré invariance $\left(\omega_{k}=\frac{41}{24}\right)$ by summing $\sim 50$ different dimensionally continued contributions.
\end{abstract}

\section{INTRODUCTION}

The problem of the gravitational interaction, within Einstein's theory, of two bodies has been revived by the realization that the most promising candidate sources for ground-based interferometric gravitational-wave detectors such as LIGO and VIRGO are binary black holes. The most significant part of the gravitational-wave signal emitted by two black holes corresponds to the last few orbits before coalescence. This makes it urgent to derive the equations of motion of two black holes with the highest possible accuracy. The equations of motion of two compact bodies (black holes or neutron stars) at the 2.5 post-Newtonian $(2.5 \mathrm{PN})$ approximation were first obtained in Refs. [1] 3 ]. [We recall that " $n \mathrm{PN}$ approximation" refers to the terms of fractional order $(v / c)^{2 n} \sim\left(G M /\left(c^{2} r\right)\right)^{n}$ in the equations of motion.] Of special importance for the following was the proof [3] that the property of "effacement" of the internal structures of (non-spinning) gravitationally interacting compact bodies implied that their equations of motions depended only on their two "Schwarzschild" masses $m_{1}, m_{2}$ up to the 5PN level. This effacement property was used to prove in [3] that the 2.5PN equations of motion of two compact objects were correctly obtained by: (i) modelling each compact object by a point-mass, i.e. a Dirac-delta-function source, and (ii) regularizing the divergencies generated by the use of delta-function sources by Riesz' analytic continuation method [4]. It was also mentioned in [5] that the correct, "effaced" equations of motion could as well be obtained by regularizing the $2.5 \mathrm{PN}$ divergencies by using dimensional regularization.

More recently the 3PN equations of motion of two point-masses have been obtained by two separate groups: [6 8] and [9.10], using different approaches, different coordinate gauges, and different regularization methods. The first group used the Arnowitt-Deser-Misner (ADM) canonical approach [11, and derived the two-body Hamiltonian corresponding to the ADM transverse-traceless (ADMTT) gauge. It regularized contact terms by Hadamard's partie finie, and divergent integrals by a combination of a Riesz-implemented Hadamard's partie finie approach with (when applicable) ordinary distribution theory [6, 12,7]. The second group worked with the harmonically relaxed Einstein equations and derived the equations of motion in a harmonic gauge. It regularized the contact terms and the divergent integrals by means of special-purpose variants of Hadamard's partie finie approach applicable to a certain class of "pseudo functions" 13.14]. In spite of the considerable efforts spent by both groups, their regularization methods are defective in two (interconnected) respects: (i) they leave undetermined a dimensionless parameter $\left(\omega_{s}\right.$, in the notation of the first group) which was shown in [15] to be crucial for determining the dynamics of the last few orbits, and (ii) they exhibit certain mathematical inconsistencies. By "mathematical inconsistency", we mean here that both regularization methods defeat the very purpose for which they were used, i.e. to uniquely define a perturbative solution of Einstein's theory which depends only on two masses $m_{1}, m_{2}$. We recall that Einstein's field equations $E_{\mu \nu} \equiv R_{\mu \nu}-\frac{1}{2} R g_{\mu \nu}=8 \pi G T_{\mu \nu}$ exhibit the consistency feature that they imply the equations of motion of the matter $\left(\nabla^{\nu} T_{\mu \nu}=0\right)$ as a consequence of the (contracted) Bianchi identity $\nabla^{\nu} E_{\mu \nu} \equiv 0$. To preserve this delicate consistency property when perturbatively solving Einstein's equations in some gauge it is necessary to use a regularization method which respects the basic properties of the algebraic and differential calculus of ordinary functions: such as the associativity, commutativity and distributivity of point-wise addition and multiplication, Leibniz's rule, Schwarz's rule $\left(\partial_{\mu} \partial_{\nu} f=\partial_{\nu} \partial_{\mu} f\right)$, integration by parts, etc. The regularization methods used up to now at the 3PN level violate at least one of the basic properties of the standard calculus (for instance, Leibniz's rule is violated both by the regularization method of [6,:] and that of [13,14]). 
We know only one regularization method which formally respects the basic properties of standard calculus: dimensional regularization [16 19]. It was invented as a way to preserve the gauge-symmetry of perturbative quantum gauge theories. Though our set up is classical, our basic consistency problem is to respect the gauge-symmetry (underlying the link between Bianchi identities and equations of motion) of perturbative general relativity. Dimensional regularization seems to be the ideal tool for generating a consistent perturbative solution of Einstein's theory depending only on $m_{1}, m_{2}$. In this letter, we shall show how to use dimensional regularization at the 3PN level in the ADM framework [20]. Our three main results will be: (i) all the pole parts $\propto(d-3)^{-1}$ (where $d$ denotes the continued space dimension) appearing in intermediate contributions cancel in the total 3PN Hamiltonian $H$, (ii) the contributions to $H$ which are quadratic in momenta are unambiguously determined by dimensional regularization to take the unique value ( $\left.\omega_{k}=41 / 24\right)$ which preserves global Poincaré invariance [8,96], and (iii) the momentum-independent contributions to $H$ are unambiguously determined and yield the following unique value for the heretofore undetermined dimensionless parameter entering 3PN dynamics:

$$
\omega_{s}=0 \text {. }
$$

\section{DIMENSIONAL CONTINUATION OF THE 3PN ADM HAMILTONIAN}

Let $D \equiv d+1$ denote the (analytically continued) space-time dimension. The ADM approach [11] uses a $d+1$ split of the coupled gravity-matter dynamics and works with the canonical pairs $\left(x_{a}^{i}, p_{a i}\right)$ and $\left(g_{i j}, \pi^{i j}\right)(i, j, k, \ldots$ denote spatial indices taking (formally) $d$ values; $a=1, \ldots, N$ labels the particles). The dimensionally continued hamiltonian and momentum constraints read (in units where $16 \pi G_{D}=1$ )

$$
\begin{aligned}
\sqrt{g} R & =\frac{1}{\sqrt{g}}\left(g_{i k} g_{j \ell} \pi^{i j} \pi^{k \ell}-\frac{1}{d-1}\left(g_{i j} \pi^{i j}\right)^{2}\right)+\sum_{a}\left(m_{a}^{2}+g_{a}^{i j} p_{a i} p_{a j}\right)^{\frac{1}{2}} \delta_{a}, \\
-2 D_{j} \pi^{i j} & =\sum_{a} g_{a}^{i j} p_{a j} \delta_{a} .
\end{aligned}
$$

Here $\delta_{a} \equiv \delta\left(\mathbf{x}-\mathbf{x}_{a}\right.$ ) (with $\int d^{d} x \delta(\mathbf{x})=1$ ), $g_{a}^{i j} \equiv g^{i j}\left(\mathbf{x}_{a}\right)$ (which will be seen to be perturbatively unambiguously defined and finite), and $D_{j}$ denotes the $d$-dimensional covariant derivative (acting on a tensor density).

The application of the ADM formalism to the gravitational dynamics of $N$ particles consists of four steps. Step (i) consists in fixing the gauge by demanding that $g_{i j}$ and $\pi^{i j}$ have the forms (ADMTT gauge)

$$
g_{i j}=A(\phi) \delta_{i j}+h_{i j}^{\mathrm{TT}}, \quad \pi^{i j}=\widetilde{\pi}^{i j}\left(V^{k}\right)+\pi_{\mathrm{TT}}^{i j},
$$

where

$$
\begin{gathered}
A(\phi) \equiv\left(1+\frac{d-2}{4(d-1)} \phi\right)^{4 /(d-2)}, \\
\widetilde{\pi}^{i j}\left(V^{k}\right) \equiv \partial_{i} V^{j}+\partial_{j} V^{i}-\frac{2}{d} \delta^{i j} \partial_{k} V^{k},
\end{gathered}
$$

and where the TT pieces $h_{i j}^{\mathrm{TT}}, \pi_{\mathrm{TT}}^{i j}$ are transverse and traceless, i.e. satisfy $\partial_{j} f_{i j}^{\mathrm{TT}}=0=\delta^{i j} f_{i j}^{\mathrm{TT}}$ with $f=h$ or $\pi$.

Step (ii) consists in solving, by a perturbative expansion, the constraints (2.1) by expressing the "longitudinal" variables $\phi$ and $\widetilde{\pi}^{i j}$ (i.e. $V^{i}$ ) in terms of the dynamical degrees of freedom $\left(x_{a}^{i}, p_{a i} ; h_{i j}^{\mathrm{TT}}, \pi_{\mathrm{TT}}^{i j}\right)$. This is done by means of a post-Newtonian (PN) expansion: $\phi=\phi_{(2)}+\phi_{(4)}+\phi_{(6)}+\phi_{(8)}+\phi_{(10)}, V^{i}=V_{(3)}^{i}+V_{(5)}^{i}+V_{(7)}^{i}$. Here, the numbers within parentheses denote the formal orders in the inverse velocity of light. E.g., $\phi_{(2)} \sim G m /\left(c^{2} r^{d-2}\right)$. [Apart from this formal use of $1 / c$ we set everywhere $c=1$.] For instance, we have

$$
\begin{aligned}
\Delta \phi_{(2)} & =-\sum_{a} m_{a} \delta_{a}, \\
\Delta \phi_{(4)} & =-\frac{1}{2} \sum_{a} \frac{\mathbf{p}_{a}^{2}}{m_{a}} \delta_{a}+\frac{d-2}{4(d-1)} \phi_{(2)} \sum_{a} m_{a} \delta_{a}, \\
\partial_{j} \widetilde{\pi}_{(3)}^{i j}(V) & \equiv \Delta V_{(3)}^{i}+\left(1-\frac{2}{d}\right) \partial_{i j} V_{(3)}^{j}=-\frac{1}{2} \sum_{a} p_{a i} \delta_{a} .
\end{aligned}
$$


The solution of Eq. 2.4c) can be written as

$$
V_{(3)}^{i}=\frac{d-2}{4(d-1)} \sum_{a} p_{a j}\left(\Delta^{-2} \delta_{a}\right)_{, i j}-\frac{1}{2} \sum_{a} p_{a i}\left(\Delta^{-1} \delta_{a}\right) .
$$

Step (iii) consists in expressing the total energy of the system $E[\phi]$ (given by the surface integral at spatial infinity of $\left.-\partial_{i} \phi\right)$ in terms of the (particle and gravitational) dynamical degrees of freedom. This yields an Hamiltonian $H\left(x_{a}^{i}, p_{a i}, h_{i j}^{\mathrm{TT}}, \pi_{\mathrm{TT}}^{i j}\right)$ which describes the coupled dynamics of matter and gravity. The fourth and final step used in the ADM approach to the problem of motion of $N$ point masses 21 23, 6] consists in: (iv) eliminating the gravitational variables $\left(h_{i j}^{\mathrm{TT}}, \pi_{\mathrm{TT}}^{i j}\right)$ by perturbatively solving their field equations (as obtained from varying the Hamiltonian derived in the previous step). This is again done by replacing $h_{i j}^{\mathrm{TT}}$ and $\pi_{\mathrm{TT}}^{i j}$ by the PN-expanded solutions of their field equations. [We are interested here in the conservative dynamics and use a time-symmetric (half-retarded half-advanced) Green function. See [22,24] for the discussion of radiation-reaction effects in the ADM approach, and 23,6,7] for the subtlety of the reduction of the higher time-derivatives appearing when solving $\left(h_{i j}^{\mathrm{TT}}, \pi_{\mathrm{TT}}^{i j}\right)$ in terms of the matter.] These PN expansions start as: $h_{i j}^{\mathrm{TT}}=h_{(4) i j}^{\mathrm{TT}}+h_{(6) i j}^{\mathrm{TT}}+\cdots, \pi_{\mathrm{TT}}^{i j}=\pi_{(5) \mathrm{TT}}^{i j}+\pi_{(7) \mathrm{TT}}^{i j}+\cdots$. Actually, the fact that the field equations for $h_{i j}^{\mathrm{TT}}, \pi_{\mathrm{TT}}^{i j}$ must be satisfied implies that it is sufficient (at $3 \mathrm{PN}$ ) to replace $\left(h_{i j}^{\mathrm{TT}}, \pi_{\mathrm{TT}}^{i j}\right)$ by their leading contributions $\left(h_{(4) i j}^{\mathrm{TT}}, \pi_{(5) \mathrm{TT}}^{i j}\right)$. These quantities satisfy the equations

$$
\begin{aligned}
\Delta h_{(4) i j}^{\mathrm{TT}} & =\left(-\sum_{a} \frac{p_{a i} p_{a j}}{m_{a}} \delta_{a}-\frac{d-2}{2(d-1)} \phi_{(2), i} \phi_{(2), j}\right)^{\mathrm{TT}}, \\
\pi_{(5) \mathrm{TT}}^{i j} & =\frac{1}{2} \dot{h}_{(4) i j}^{\mathrm{TT}}+\frac{d-2}{d-1}\left(\phi_{(2)} \widetilde{\pi}_{(3)}^{i j}\right)^{\mathrm{TT}} .
\end{aligned}
$$

The superscripts TT in Eqs. (2.6) denote the application to a second rank tensor of the $d$-dimensional (spatially nonlocal) TT-projection operator.

The matter Hamiltonian obtained after elimination of the gravitational variables can be simplified by using many integration by parts allowing one to successively eliminate $\phi_{(8)}, \phi_{(6)}, V_{(7)}^{i}$ and $V_{(5)}^{i}$ by using the elliptic equations they must satisfy. [Some details of this elimination process will be discussed in [20].] For convenience, we have performed this elimination procedure in a form closely parallel to the one performed (when $d=3$ ) in [7]. Finally, the dimensional continuation of the matter-reduced dimensionally continued 3PN Hamiltonian reads (we do not write the $d$-dimensional versions of the non-problematic $1 \mathrm{PN}$ and $2 \mathrm{PN}$ Hamiltonians)

$$
H_{3 \mathrm{PN}}=-\frac{5}{128} \sum_{a} \frac{\left(\mathbf{p}_{a}^{2}\right)^{4}}{m_{a}^{7}}+\int d^{d} x\left(h_{1}+h_{2}+h_{3}\right)
$$

where

$$
\begin{aligned}
h_{1}= & \left(\frac{(4-d)(d-2)^{2}}{64(d-1)^{3}} S_{(4)} \phi_{(2)}^{2}-\frac{(4-d)(d-2)}{16(d-1)^{2}} S_{(4)} \phi_{(4)}\right) \sum_{a} m_{a} \delta_{a} \\
& +\left(-\frac{d(d+2)(3 d-4)}{192(d-1)^{3}} \phi_{(2)}^{3}+\frac{(d+2)(3 d-4)}{16(d-1)^{2}} \phi_{(2)} \phi_{(4)}+\frac{(d-4)(d+2)}{32(d-1)^{2}} S_{(4)} \phi_{(2)}\right) \sum_{a} \frac{\mathbf{p}_{a}^{2}}{m_{a}} \delta_{a} \\
& +\left(-\frac{d(d+6)}{64(d-1)^{2}} \phi_{(2)}^{2}+\frac{d}{8(d-1)} \phi_{(4)}-\frac{4-d}{32(d-1)} S_{(4)}\right) \sum_{a} \frac{\left(\mathbf{p}_{a}^{2}\right)^{2}}{m_{a}^{3}} \delta_{a}-\frac{d+4}{32(d-1)} \phi_{(2)} \sum_{a} \frac{\left(\mathbf{p}_{a}^{2}\right)^{3}}{m_{a}^{5}} \delta_{a} \\
& +\frac{4-d}{4(d-1)} S_{(4)}\left(\widetilde{\pi}_{(3)}^{i j}\right)^{2}-\frac{3 d-4}{2(d-1)} \phi_{(4)}\left(\widetilde{\pi}_{(3)}^{i j}\right)^{2} \\
& +\frac{(4-d)(d-2)}{4(d-1)^{2}} \phi_{(2), i} S_{(4), j} h_{(4) i j}^{\mathrm{TT}}-\frac{(d-2)(2 d-3)}{2(d-1)^{2}} \phi_{(2), i} \phi_{(4), j} h_{(4) i j}^{\mathrm{TT}}+\frac{1}{4} h_{(4) i j}^{\mathrm{TT}} \sum_{a} \frac{\mathbf{p}_{a}^{2} p_{a i} p_{a j}}{m_{a}^{3}} \delta_{a} \\
& -\left(\left(\phi_{(2)} \widetilde{\pi}_{(3)}^{i j}\right)^{\mathrm{TT}}\right)^{2}-\frac{d-2}{d-1}\left(\phi_{(2)} \tilde{\pi}_{(3)}^{i j}\right)^{\mathrm{TT}} \dot{h}_{(4) i j}^{\mathrm{TT}}-\frac{1}{4}\left(\dot{h}_{(4) i j}^{\mathrm{TT}}\right)^{2},
\end{aligned}
$$




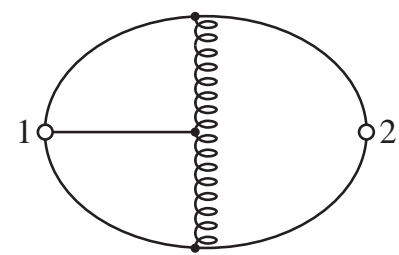

FIG. 1. "Three-loop diagram" representing the contribution (of order $G^{4} m_{1}^{3} m_{2}^{2}$ ) proportional to $\int d^{d} x \phi_{1} \partial_{k} h_{(40) i j}^{\mathrm{TT}} \partial_{k} h_{(40) i j}^{\mathrm{TT}}$ in the Hamiltonian, where $h_{(40) i j}^{\mathrm{TT}} \equiv-2 c(d) \Delta^{-1}\left(\partial_{i} \phi_{1} \partial_{j} \phi_{2}\right)^{\mathrm{TT}}$. The solid lines represent the "propagator" $\Delta^{-1}$ of $\phi$, the helicoidal lines represent the "propagator" $\left(\Delta^{-1}\right)^{\mathrm{TT}}$ of $h_{(4) i j}^{\mathrm{TT}}$, and the circles labelled 1 and 2 denote the sources $m_{1} \delta_{1}$ and $m_{2} \delta_{2}$.

$$
\begin{aligned}
h_{2}= & \left(\frac{(d-2)^{4}}{128(d-1)^{4}} \phi_{(2)}^{4}-\frac{(d-2)^{3}}{8(d-1)^{3}} \phi_{(2)}^{2} \phi_{(4)}+\frac{(d-2)^{2}}{4(d-1)^{2}} \phi_{(4)}^{2}\right) \sum_{a} m_{a} \delta_{a}, \\
h_{3}= & \frac{(3 d-4)(3 d-2)}{16(d-1)^{2}} \phi_{(2)}^{2}\left(\widetilde{\pi}_{(3)}^{i j}\right)^{2}+2\left(V_{(3), i}^{k} V_{(3), j}^{k}-V_{(3), k}^{i} V_{(3), k}^{j}-\frac{4}{d} V_{(3), j}^{i} V_{(3), k}^{k}\right) h_{(4) i j}^{\mathrm{TT}} \\
& +4 V_{(3)}^{i} V_{(3), j}^{k} h_{(4) i j, k}^{\mathrm{TT}}+\frac{(d-2)(3 d-4)}{8(d-1)^{3}} \phi_{(2)} \phi_{(2), i} \phi_{(2), j} h_{(4) i j}^{\mathrm{TT}} \\
& -\frac{d+2}{4(d-1)}\left(\phi_{(2)} h_{(4) i j}^{\mathrm{TT}}\right)_{, k} S_{(4) i j, k}-\frac{1}{2(d-1)}\left(\phi_{(2)} h_{(4) i j}^{\mathrm{TT}}\right)_{, k} h_{(4) i j, k}^{\mathrm{TT}} .
\end{aligned}
$$

The 3PN Hamiltonian (2.7) is expressed in terms of the following quantities: $\phi_{(2)}$ defined by Eq. (2.4a), $\phi_{(4)}$ defined by Eq. (2.4b), $V_{(3)}^{i}$ defined by Eq. (2.5), $\tilde{\pi}_{(3)}^{i j} \equiv \tilde{\pi}^{i j}\left(V_{(3)}^{k}\right)$ defined by Eq. (2.3b), $h_{(4) i j}^{\mathrm{TT}}$ defined by Eq. (2.6a), as well as $S_{(4)}$ and $S_{(4) i j}$ defined by the following equations

$$
S_{(4)} \equiv \Delta^{-1} \sum_{a} \frac{\mathbf{p}_{a}^{2}}{m_{a}} \delta_{a}, \quad S_{(4) i j} \equiv \Delta^{-1} \sum_{a} \frac{p_{a i} p_{a j}}{m_{a}} \delta_{a}
$$

Using the natural (propagator)*(source) structure of each building block of the Hamiltonian $H$, we can represent $H$ by a sum of diagrams. For instance the last term in Eq. (2.80) gives rise (after expanding it in powers of $m_{1}$ and $\left.m_{2}\right)$ to several diagrams, one of which $\left(\propto m_{1}^{3} m_{2}^{2}\right)$ is represented (for illustration) in Fig. 1. Note that this diagram contains three (classical) loops. More generally the most nonlinear (momentum-independent) contributions to $H_{n \mathrm{PN}}$ contain $n$ loops.

\section{FINITENESS OF $H_{\mathrm{ADM}}(d)$ AS $d \rightarrow 3$}

The perturbative consistency of dimensional regularization [19] ensures that the formal $d$-dimensional PN-expanded solution $g_{\mu \nu}\left(x^{\lambda}\right)$ given in Section II will directly generate, when $d$ is analytically continued up to $d=3$, a 3 -dimensional solution of Einstein's theory if it is finite as $d \rightarrow 3$, i.e. if no poles proportional to $(d-3)^{-1}$ arise when $d \rightarrow 3$. One can analytically find out all the potentially "dangerous" terms (those which might give rise to a pole) in $H_{3 \mathrm{PN}}, \mathrm{Eq}$. (2.7), by the following analysis. First, the $d$-dimensional solution of the basic equation $\Delta u_{a}=-\delta_{a}$ is $u_{a} \equiv-\Delta^{-1} \delta_{a}=k r_{a}^{2-d}$, where $k \equiv \Gamma\left(\frac{d-2}{2}\right) /\left(4 \pi^{\frac{d}{2}}\right)$ and $r_{a} \equiv\left|\mathbf{x}-\mathbf{x}_{a}\right|$. [Though $k$ depends on $d$ we shall factor the various powers of $k$ entering each contribution to $H_{3 \mathrm{PN}}$ without ever expanding its dependence on $d$ as $d \rightarrow 3$.] This allows one to write down explicitly $\phi_{(2)}, \phi_{(4)}, S_{(4)}$, and $S_{(4) i j}$. The explicit expression of $V_{(3)}^{i}$ is then obtained by iterating the Poisson operator $\Delta^{-1}$ using $\Delta^{-1} r_{a}^{\lambda}=r_{a}^{\lambda+2} /((\lambda+2)(\lambda+d))$.

These results suffice to write down the part of $h_{(4) i j}^{\mathrm{TT}}$, Eq. (2.6a), whose source is $-m_{a}^{-1} p_{a i} p_{a j} \delta_{a}$. We denote this part as $h_{(42) a i j}^{\mathrm{TT}}$ (with $\left.a=1,2\right)$. The part of $h_{(4) i j}^{\mathrm{TT}}$ which does not depend on momenta will be denoted by $h_{(40) i j}^{\mathrm{TT}}$. One can control its crucial "ultra violet" (UV) behaviour as $r_{a} \rightarrow 0$ (which suffices to analyze the potentially dangerous terms) in the following way. First, let $c(d) \equiv(d-2) /(2(d-1))$ so that the source term for $h_{(40) i j}^{\text {TT }}$ is the TT projection of $-c(d) \sigma_{i j}\left(\phi_{(2)}, \phi_{(2)}\right)$ where $\sigma_{i j}(\phi, \psi) \equiv \partial_{i} \phi \partial_{j} \psi$. Inserting the solution $\phi_{(2)}=m_{1} u_{1}+m_{2} u_{2}$ into $\sigma_{i j}$, and applying the TT operator (which projects to zero $\sigma_{i j}\left(u_{1}, u_{1}\right)$ and $\sigma_{i j}\left(u_{2}, u_{2}\right)$ ), yields $h_{(40) i j}^{\mathrm{TT}}=-2 c(d) m_{1} m_{2} \Delta^{-1}\left(\sigma_{i j}\left(u_{1}, u_{2}\right)\right)^{\mathrm{TT}}$. 
Operating by parts under the TT operator further yields $h_{(40) i j}^{\mathrm{TT}}=+2 c(d) m_{1} m_{2} \Delta^{-1}\left(u_{1} \partial_{i j} u_{2}\right)^{\mathrm{TT}}$. It is then easily proven that, near $\mathbf{x}_{1}, h_{(40) i j}^{\mathrm{TT}}$ is the sum of a regular $\left(C^{\infty}\right)$ function and of a singular expansion in powers of $r_{1}$ obtained by inserting in the source term $\propto\left(u_{1} \partial_{i j} u_{2}\right)^{\mathrm{TT}}$ the Taylor expansion of $\partial_{i j} u_{2}(\mathbf{x})$ near $\mathbf{x}=\mathbf{x}_{1}$, and applying the operator $\Delta^{-1}$ to each term of the expansion.

Using this local UV analysis we studied the convergence, near, say, $\mathbf{x}_{1}$, of the integral giving $H_{\mathrm{ADM}}$. The conclusion is that poles $\propto(d-3)^{-1}$ can arise only from the third integrand $h_{3}$, Eq. (2.8c). More precisely, one finds that, when expanding each field entering $h_{3}$ in powers of the masses $m_{1}$ and $m_{2}$, and then expanding each partial integrand in powers of $r_{1}$ there arise ten (and only ten) separate terms $T_{A}, A=1, \ldots, 10$, giving rise to poles as $d \rightarrow 3$. These pole-generating partial integrands have all the structure (for the poles generated by the integration near $r_{1}=0$ )

$$
\begin{aligned}
T_{A}= & k^{4} m_{1} m_{2} r_{1}^{6-3 d}\left(c_{A 1} D\left(p_{1}, p_{1}\right)+c_{A 2}\left(n_{1} \cdot p_{1}\right) D\left(n_{1}, p_{1}\right)\right. \\
& \left.+c_{A 3}\left(n_{1} \cdot p_{1}\right)^{2} D\left(n_{1}, n_{1}\right)+c_{A 4} p_{1}^{2} D\left(n_{1}, n_{1}\right)\right),
\end{aligned}
$$

where $c_{A 1}, \ldots, c_{A 4}$ are $d$-dependent coefficients and where $D_{i j} \equiv\left[\partial_{i j} r_{2}^{2-d}\right]_{\mathbf{x}=\mathbf{x}_{1}}, D(p, q) \equiv D_{i j} p^{i} q^{j}, n_{1}^{i} \equiv r_{1}^{-1}\left(x^{i}-x_{1}^{i}\right)$, and $n_{1} \cdot p_{1} \equiv n_{1}^{i} p_{1 i} \equiv \delta_{i j} n_{1}^{i} p_{1}^{j}$. These ten terms arise from the terms in the mass-expanded version of $h_{3}$ with the respective structures: $\phi_{1} \phi_{2}\left(\partial V_{1}\right)^{2}, \partial V_{1} \partial V_{1} h_{(40)}^{\mathrm{TT}}, V_{1} \partial V_{1} \partial h_{(40)}^{\mathrm{TT}}, \phi_{2} \partial \phi_{1} \partial \phi_{1} h_{(42) 1}^{\mathrm{TT}}, \phi_{1} \partial \phi_{2} \partial \phi_{1} h_{(42) 1}^{\mathrm{TT}}, \phi_{1} \partial h_{(42) 1}^{\mathrm{TT}} \partial h_{(40)}^{\mathrm{TT}}$, $\partial S_{1} \partial \phi_{1} h_{(40)}^{\mathrm{TT}}, \partial S_{1} \phi_{1} \partial h_{(40)}^{\mathrm{TT}}, \partial \phi_{1} \partial h_{(42) 1}^{\mathrm{TT}} h_{(40)}^{\mathrm{TT}}$, and $\partial \phi_{1} h_{(42) 1}^{\mathrm{TT}} \partial h_{(40)}^{\mathrm{TT}}$. Here we have suppressed, for readibility, the PN indices (2) (on $\phi$ ), (3) (on $V$ ) and (4) (on $S$ ), but we left the indices (40) and (42) on $h^{\mathrm{TT}}$. The explicit labels 1 or 2 refer to their sources $\propto \delta_{1}$ or $\delta_{2}$. Each term $T_{A}$ carries its full coefficient, as arising from the successive (mass-expanded and Taylor-expanded) terms in $h_{3}$, Eq. (2.8d).

One then computes the "local" contribution (in a ball around $\mathbf{x}_{1}$ of radius $\ell_{1}$, with $0<\ell_{1} \ll r_{12} \equiv\left|\mathbf{x}_{1}-\mathbf{x}_{2}\right|$ ) of each "dangerous" term $T_{A}$. After averaging (in a $d$-dimensional sense) over angles, and integrating over the radius $r_{1}$, we find that each "dangerous" term locally yields, when $d \rightarrow 3$, the following "pole" contribution to the Hamiltonian (2.7)

$$
H_{A}^{\mathrm{loc}}(d)=-\frac{1}{2} \Omega_{d} k^{4} m_{1} m_{2} D\left(p_{1}, p_{1}\right) \ell_{1}^{-2(d-3)} \frac{c_{A}(d)}{d-3},
$$

where $\Omega_{d}$ is the area of the unit sphere in $d$ dimensions, and where we defined

$$
c_{A}(d) \equiv c_{A 1}(d)+\frac{c_{A 2}(d)}{d}+\frac{2 c_{A 3}(d)}{d(d+2)} .
$$

We have explicitly computed the $10 \times 4=40 d$-dependent coefficients $c_{A 1}(d), \ldots, c_{A 4}(d)$ as well as the 10 angularaveraged coefficients $c_{A}(d)$. Note that the value of each of these coefficients delicately depends on having correctly derived the $d$-dependent coefficients appearing in the Hamiltonian, as well as all the $d$-dependent factors arising from the $\Delta^{-1}$ and TT operators and from the angular averaging. The results are listed in Table I, each coefficient being expanded in powers of $\varepsilon \equiv d-3$, e.g. $c_{A}(d)=c_{A}(3)+\varepsilon c_{A}^{\prime}(3)+\mathcal{O}\left(\varepsilon^{2}\right)$. A first important result from Table I is that, as one checks, the sum of the ten 3 -dimensional averaged coefficients $c_{A}(3)$ exactly vanishes:

$$
\sum_{A=1}^{10} c_{A}(3)=0 .
$$

In view of Eq. (3.2) this proves that the total pole part, as $d \rightarrow 3$, of $H(d)$ vanishes. In other words, dimensional continuation up to $d=3$ gives a unique, finite value for $H_{3 \mathrm{PN}}^{\mathrm{ADM}}$. [This result confirms the finding of [6, ]6] that all the "Riesz poles" appearing in the Riesz-based implementation of Hadamard's partie finie finally cancel. However, this finding was not sufficient for being able to unambiguously compute the "regularized" value of $H_{3 \mathrm{PN}}^{\mathrm{ADM}}$.]

\section{DIMENSIONAL-CONTINUATION DETERMINATION OF THE HAMILTONIAN}

Having shown that $H_{3 \mathrm{PN}}^{\mathrm{ADM}}(d)$ has a unique, finite value as $d \rightarrow 3$, it remains the crucial task of explicitly computing this finite value. Let us first focus on the $h_{3}$-generated part of the Hamiltonian, i.e. on $H_{3}(d) \equiv \int d^{d} x h_{3}(d)$, with $h_{3}(d)$ given by Eq. (2.8d), and on the finite limit: $\lim _{d \rightarrow 3} H_{3}(d)$. Let us compute the difference $\Delta H_{3} \equiv \lim _{d \rightarrow 3} H_{3}(d)-H_{3}^{\text {DJS }}$, where $H_{3}^{\text {DJS }} \equiv \mathrm{RH}\left[\int d^{3} x h_{3}^{\text {DJS }}\right]$ is the Riesz-implemented Hadamard regularization (RH), as defined in [6,12,7], of the 
TABLE I. Expansion in powers of $\varepsilon \equiv d-3$ of the $d$-dependent coefficients $c_{A 1}(d), \ldots, c_{A 4}(d)$ and $c_{A}(d)$ entering the 10 "dangerous" terms $T_{A}, A=1, \ldots, 10$.

\begin{tabular}{|c|c|c|c|c|c|}
\hline$A$ & $c_{A 1}$ & $c_{A 2}$ & $c_{A 3}$ & $c_{A 4}$ & $c_{A}$ \\
\hline 1 & 0 & 0 & $\frac{315}{1024}+\frac{3711}{4096} \varepsilon$ & $\frac{315}{2048}+\frac{267}{1024} \varepsilon$ & $\frac{21}{512}+\frac{1013}{10240} \varepsilon$ \\
\hline 2 & $-\frac{13}{256}-\frac{263}{1536} \varepsilon$ & $-\frac{3}{128}-\frac{47}{256} \varepsilon$ & $\frac{15}{512}+\frac{33}{256} \varepsilon$ & $\frac{5}{512}+\frac{235}{3072} \varepsilon$ & $-\frac{7}{128}-\frac{1649}{7680} \varepsilon$ \\
\hline 4 & 0 & 0 & $\frac{15}{512}+\frac{191}{2048} \varepsilon$ & $-\frac{5}{512}-\frac{57}{2048} \varepsilon$ & $\frac{1}{256}+\frac{53}{5120} \varepsilon$ \\
\hline 5 & 0 & $-\frac{5}{64}-\frac{37}{256} \varepsilon$ & $-\frac{5}{128}-\frac{57}{512} \varepsilon$ & $\frac{5}{128}+\frac{37}{512} \varepsilon$ & $-\frac{1}{32}-\frac{33}{640} \varepsilon$ \\
\hline 8 & $-\frac{65}{768}-\frac{161}{1536} \varepsilon$ & $\frac{25}{384}+\frac{5}{768} \varepsilon$ & $\frac{5}{1536}+\frac{17}{3072} \varepsilon$ & $-\frac{35}{1536}+\frac{11}{3072} \varepsilon$ & $-\frac{1}{16}-\frac{7}{64} \varepsilon$ \\
\hline 9 & $-\frac{13}{3072}-\frac{23}{1536} \varepsilon$ & $-\frac{19}{1536}-\frac{49}{768} \varepsilon$ & $\frac{7}{6144}-\frac{11}{4096} \varepsilon$ & $\frac{23}{6144}+\frac{257}{12288} \varepsilon$ & $-\frac{21}{2560}-\frac{5423}{153600}$ \\
\hline 10 & $\frac{13}{3072}+\frac{5}{768} \varepsilon$ & $\frac{19}{1536}+\frac{5}{128} \varepsilon$ & $-\frac{7}{6144}+\frac{61}{12288} \varepsilon$ & $-\frac{23}{6144}-\frac{55}{4096} \varepsilon$ & $\frac{21}{2560}+\frac{2903}{153600} \varepsilon$ \\
\hline
\end{tabular}

3-dimensional integral of the integrand $h_{3}^{\text {DJS }}$ given by Eq. (A9c) of $\left[77\right.$. Note that each term in $h_{3}(d)$, Eq. (2.8c), tends, when $d \rightarrow 3$, to a corresponding term (with the same coefficient in the limit) in $h_{3}^{\text {DJS }}$. The comparison between the two integrals $H_{3}(d)$ and $H_{3}^{\text {DJS }}$ is best done by: (i) separating the full space integral into two local integrals (restricted to balls of radii $\ell_{1}$ and $\ell_{2}$ around $\mathbf{x}_{1}$ and $\mathbf{x}_{2}$ ) and a global integral over the rest of space, and (ii) decomposing each term of each integrand into a regular part (which is absolutely convergent in $d=3$ ) and a singular part (which is not absolutely convergent in $d=3$ ) made of a finite number of terms of the form (say, near $\left.\mathbf{x}_{1}\right) r_{1}^{-\lambda} C_{i_{1} \cdots i_{k}} n_{1}^{i_{1}} \cdots n_{1}^{i_{k}}$. It is then seen that the difference $\Delta H_{3}$ is simply given by the difference between $\lim _{d \rightarrow 3} H_{3}^{\text {loc sing }}(d)$ and $\mathrm{RH}\left[H_{3 \mathrm{DJS}}^{\text {loc sing }}\right]$, involving only the local $\left(r_{1}<\ell_{1}\right.$ or $\left.r_{2}<\ell_{2}\right)$ integrals of the singular parts of the integrands. Next, one checks that all local singularities which do not correspond to poles in $d-3$ are given, in the limit $d \rightarrow 3$, the same regularized values in both regularization methods. However, the situation is different for the local ("logarithmic") singularities $\propto r_{1}^{-3}$ in $d=3$, which give rise to poles in $d \neq 3$. The complete list of these "dangerous" local singularities has been given above: they are the ten terms $T_{A}$ listed in Table I. [Note that they are all quadratic in $p_{1}$. Here, we focus on the UV behaviour near $\mathbf{x}_{1}$; one should add the similar terms obtained by exchanging $1 \leftrightarrow 2$.] After averaging over angles, the RH regularization of the $d=3$ limit of each local singular integral is $\propto c_{A}(3) \ln \left(\ell_{1} / s_{1}\right)$ where $s_{1}$ is the scale entering the definition of the RH operation. After summing over $A=1, \ldots, 10$, the result (3.4) shows that $\mathrm{RH}\left(H_{3 \mathrm{DJS}}^{\text {loc sing }}\right)=0$. On the other hand, dimensional continuation gives for the value of $H_{3}^{\text {loc sing }}(d)$ the sum over $A$ of the values (3.2). Finally, we conclude, using $c_{A}(3+\varepsilon)=c_{A}(3)+\varepsilon c_{A}^{\prime}(3)+\mathcal{O}\left(\varepsilon^{2}\right)$, Eq. (3.4), and the 3-dimensional limits $\Omega_{3}=4 \pi$, $k(d=3)=1 /(4 \pi)$, that $\left(r_{12} \equiv\left|\mathbf{x}_{1}-\mathbf{x}_{2}\right|\right)$

$$
\Delta H_{3} \equiv \lim _{d \rightarrow 3} H_{3}(d)-H_{3}^{\text {DJS }}=-\frac{1}{2}\left(\sum_{A=1}^{10} c_{A}^{\prime}(3)\right) \frac{m_{1} m_{2}}{(4 \pi)^{3}} p_{1 i} p_{1 j} \partial_{i j} \frac{1}{r_{12}}+1 \leftrightarrow 2 .
$$

We have similarly evaluated $\Delta H_{i} \equiv \lim _{d \rightarrow 3} H_{i}(d)-H_{i}^{\text {DJS }}$, for $i=1,2$, where $H_{i}(d) \equiv \int d^{d} x h_{i}(d)$ and $H_{i}^{\text {DJS }} \equiv$ $\mathrm{RH}\left[\int d^{3} x h_{i}^{\mathrm{DJS}}\right]$. Here the formal expressions of $h_{1}(d)$ and $h_{2}(d)$ do not seem to correspond to the expressions of $h_{1}^{\mathrm{DJS}}$, 
$h_{2}^{\text {DJS }}$ given in Eqs. (A9a), (A9b) of [7]. However, we can use the properties of dimensional regularization to transform $h_{1}(d)$ and $h_{2}(d)$. We have checked that, by using suitable integration by parts (which are allowed in dimensional continuation), one could transform $h_{i}(d), i=1,2$, into new expressions $h_{i}^{\prime}(d)$ which are term-by-term similar to $h_{i}^{\text {DJS }}$, in the same sense that $h_{3}(d)$ was similar to $h_{3}^{\text {DJS }}$. An analysis (using the tools given above) of the UV behaviour of $h_{i}^{\prime}(d)$ then shows that they contain no "dangerous" terms $\mathcal{O}\left(r_{1}^{-\lambda(d)}\right)$ with $\lambda(3)=3$. The reasoning of the previous section then shows that $\Delta H_{1}=\Delta H_{2}=0$.

Finally, we conclude that the finite value of the total Hamiltonian is given by: $\lim _{d \rightarrow 3} H_{3 \mathrm{PN}}^{\mathrm{ADM}}(d)=H_{3 \mathrm{PN}}^{\mathrm{DJS}}+\Delta H_{3}$ with $\Delta H_{3}$ given by Eq. (4.1). The two main consequences of this result concern the two dimensionless parameters that were left undetermined by the original Riesz-Hadamard regularization of the 3PN Hamiltonian [6]: $\omega_{k}$ and $\omega_{s}$.

First, from the definition (see, e.g., Eq. (13) of [8]) of the dimensionless "kinetic" parameter $\omega_{k}$ and the fact that $H_{3}^{\text {DJS }}$ corresponds, by convention, to $\omega_{k}=0$, the result (4.1) is equivalent to saying that dimensional continuation uniquely determines the value of $\omega_{k}$ to be (using $(4 \pi)^{-1}=4 G$ )

$$
\omega_{k}^{\text {dim reg }}=64 \sum_{A=1}^{10} c_{A}^{\prime}(3) .
$$

The sum of the $c_{A}^{\prime}(3)$ read from Table I is checked to be $\frac{41}{1536}$. The dimensional continuation prediction (4.2) therefore yields $\omega_{k}^{\operatorname{dim} r e g}=\frac{41}{24}$. As shown in [8] (see also [9,10]) this value is the unique, correct value which ensures that the regularized Hamiltonian admits a nonlinear realization of global Poincaré invariance. We find it truly remarkable that the use of dimensional regularization in the ADM formalism (which violates from the start manifest Poincaré invariance by splitting space and time and by using a non Poincaré-invariant gauge) uniquely predicts, after a long calculation involving many intermediate complicated $d$-dependent coefficients and finally summing 50 different contributions of Table I (indeed $c_{A}^{\prime}(3)$ is a combination of $c_{A 1}^{\prime}, c_{A 2}^{\prime}, c_{A 3}^{\prime}, c_{A 2}$, and $c_{A 3}$ for $A=1, \ldots, 10$ ), the unique, physically correct value of $\omega_{k}$. We interpret this as a very strong confirmation of the mathematical (and physical) consistency of dimensional regularization, and, in particular, of its property of perturbatively respecting gauge symmetry (i.e., in our case, diffeomorphism invariance).

Second, as $\Delta H_{3}$ affects only the part of the Hamiltonian which is quadratic in the momenta, we conclude that the heretofore undetermined "static parameter" $\omega_{s}$ is simply: $\omega_{s}=0$ (as it was in the "reference" Hamiltonian $H^{\text {DJS }}$. . Note that this simple conclusion was obtained after a detailed analysis of the UV behaviour of all the terms in (2.7). In this analysis, the ADMTT gauge played a very useful role in suppressing many of the stronger UV divergencies that occur in a harmonic gauge. For instance the contribution $I_{1}(d)=\int d^{d} x \phi_{1} \partial_{k} h_{(40) i j}^{\mathrm{TT}} \partial_{k} h_{(40) i j}^{\mathrm{TT}}$ where $h_{(40) i j}^{\mathrm{TT}} \equiv-c(d) \Delta^{-1}\left(\partial_{i} \phi \partial_{j} \phi\right)^{\mathrm{TT}}$, which corresponds to the diagram depicted in Fig. 1, turns out to be completely "non dangerous" (and even locally integrable), while its harmonic-gauge analog would contain $U_{i j} \equiv-c(d) \Delta^{-1}\left(\partial_{i} \phi \partial_{j} \phi\right)$ instead of $h_{(40) i j}^{\mathrm{TT}}$ and would give rise to several (gauge-spurious) "dangerous" integrals generating pole parts (and logarithms in $d=3$ ). [The existence of poles at 3PN, in harmonic coordinates, was first mentioned in [3] and has been confirmed by the explicit results of [10].] Let us also note that dimensional regularization leads to a very simple and consistent treatment of "contact terms". Starting from the best available definition of dimensional regularization, which is in the Fourier domain (e.g. [19]), we have checked that the value of, e.g., $I_{2}(d)=\int d^{d} x \phi^{4}(\mathbf{x}) \delta_{1}$ is $\phi^{4}\left(\mathbf{x}_{1}\right)=\left(m_{2} u_{2}\left(\mathbf{x}_{1}\right)\right)^{4}$. This simple "threading" property of contact terms extends to all the perturbative contact terms arising when formally solving Einstein's equations. Here, dimensional regularization differs from some variants of Hadamard's partie finie regularization in which $\operatorname{Pf}\left(\phi^{4}\right) \neq(\operatorname{Pf}(\phi))^{4}$.

To conclude this letter let us mention some of the physical applications of our results. The most important is that our unique determination of the last missing parameter in the 3PN Hamiltonian allows one to make full use of the 3PN accuracy and, in particular, to analytically estimate the characteristics of the last stable orbits (LSO) of binary black holes. For instance, using the best available PN-resummation method, namely the (Padé-resummed) effective one-body approach [25,15,26, one finds that the LSO binding energy of two non-spinning equal-mass black holes is $1.67 \%\left(m_{1}+m_{2}\right) c^{2}$. More generally, our work allows one to extend to 3PN the approach proposed in [25], namely to start the numerical simulation of the coalescence of two black holes (without or with spin [26]) just after they cross their LSO, so that they are less than one orbit away from coalescence.

Our work contains another important lesson for numerical relativity: our dimensional-continuation calculation of $H_{3}$ and $\omega_{k}$ shows (as a corollary) that the "conformally flat" truncation (i.e. setting $h_{i j}^{\mathrm{TT}}$ to zero) which is of general use in numerical relativity, is "inconsistent" in that it ruins the delicate cancellation of poles and leads to a formally infinite 3PN Hamiltonian. [In addition, it violates global Poincaré invariance.] As the physical meaning of the $3 \mathrm{PN}$ pole cancellation is that the perturbative algorithm unambiguously determines the unique, physically correct solution of Einstein's equations depending on $m_{1}$ and $m_{2}$ which matches two black holes [20], the lack of uniqueness associated to the arbitrariness in subtracting the 3PN pole generated by the conformally flat truncation probably 
explains why current initial data simulations [27,28] significantly differ from the resummed analytical estimates of LSO characteristics.

Finally, our work having convincingly established the perturbative consistency of dimensional regularization, we strongly recommend to use it to uniquely determine the 3PN contribution to the gravitational wave flux, whose determination might otherwise be marred by spurious ambiguities. Let us also mention that it would be nice to independently confirm our results by two different calculations: (i) to use dimensional continuation, instead of a variant of Hadamard regularization [13, 14, to compute the 3PN dynamics in harmonic coordinates, and (ii) to use dimensional continuation to recompute $\omega_{s}$ by studying the motion of a test mass around a Schwarzschild black hole to the first "post-test-mass" approximation $(\mathcal{O}(\mu / M)$ corrections).

\section{ACKNOWLEDGMENTS}

This work was supported in part by the KBN Grant No. 5 P03B 03420 (to P.J.).

[1] T. Damour and N. Deruelle, Phys. Lett. 87A, 81 (1981).

[2] T. Damour, C. R. Séances Acad. Sci., Ser. 2 294, 1355 (1982).

[3] T. Damour, in Gravitational Radiation, edited by N. Deruelle and T. Piran (North-Holland, Amsterdam, 1983 ), pp. 59-144.

[4] M. Riesz, Acta Math. 81, 1 (1949).

[5] T. Damour, C. R. Séances Acad. Sci., Ser. A 291, 227 (1980).

[6] P. Jaranowski and G. Schäfer, Phys. Rev. D 57, 7274 (1998); 63, 029902(E) (2001).

[7] T. Damour, P. Jaranowski, and G. Schäfer, Phys. Rev. D 62, 044024 (2000).

[8] T. Damour, P. Jaranowski, and G. Schäfer, Phys. Rev. D 62, 021501(R) (2000); 63, 029903(E) (2001).

[9] L. Blanchet and G. Faye, Phys. Lett. A 271, 58 (2000).

[10] L. Blanchet and G. Faye, Phys. Rev. D 63, 062005 (2001).

[11] R. Arnowitt, S. Deser, and C. W. Misner, in Gravitation: An Introduction to Current Research, edited by L. Witten (Wiley, New York, 1962), pp. 227-265.

[12] P. Jaranowski, in Mathematics of Gravitation. Part II. Gravitational Wave Detection, edited by A. Królak, Banach Center Publications (Institute of Mathematics, Polish Academy of Sciences, Warszawa, 1997), Vol. 41, Part II, pp. 55-63.

[13] L. Blanchet and G. Faye, J. Math. Phys. 41, 7675 (2000).

[14] L. Blanchet and G. Faye, gr-qc/0006100.

[15] T. Damour, P. Jaranowski, and G. Schäfer, Phys. Rev. D 62, 084011 (2000).

[16] G. 't Hooft and M. Veltman, Nucl. Phys. B44, 189 (1972).

[17] C. G. Bollini and J. J. Giambiagi, Phys. Lett. 40B, 566 (1972).

[18] P. Breitenlohner and D. Maison, Comm. Math. Phys. 52, 11 (1977).

[19] J. Collins, Renormalization (Cambridge University Press, Cambridge, 1984).

[20] More details will be given in: T. Damour, P. Jaranowski, and G. Schäfer, in preparation.

[21] T. Ohta, H. Okamura, T. Kimura, and K. Hiida, Progr. Theor. Phys. 51, 1598 (1974).

[22] G. Schäfer, Ann. Phys. (N.Y.) 161, 81 (1985).

[23] T. Damour and G. Schäfer, Gen. Relativ. Gravit. 17, 879 (1985).

[24] P. Jaranowski and G. Schäfer, Phys. Rev. D 55, 4712 (1997).

[25] A. Buonanno and T. Damour, Phys. Rev. D 59, 084006 (1999); 62, 064015 (2000).

[26] T. Damour, gr-qc/0103018.

[27] T. W. Baumgarte, Phys. Rev. D 62, 024018 (2000).

[28] J. Baker et al., gr-qc/0102037. 